\title{
Comparative analysis of UAV RS and traditional cotton leaf falling evaluation methods
}

\author{
Yuzi Zhang ${ }^{1,2}$, Wenhua $\mathrm{Li}^{1}$, Weixu Ran ${ }^{1}$, Jian Gu${ }^{1}$, Yubin Lan ${ }^{1,2^{*}}$ \\ (1. College of Agricultural Engineering and Food Science, Shandong University of Technology, Shandong Provincial Engineering \\ Technology Research Center for Agricultural Aviation Intelligent Equipment, Zibo 255022, Shandong, China; \\ 2. Zibo Agricultural and Rural Service Center, Zibo 255022, Shandong, China;)
}

\begin{abstract}
Evaluating the spraying effect of defoliant is necessary condition to facilitate mechanized cotton harvesting. However, traditional method to evaluate the effect of spraying defoliation by manually calculating the number of cotton defoliation will produce large statistical error and increase the labor cost. Low altitude remote sensing provides accurate and timely estimation of biophysical parameters, such as leaf area index (LAI), crop growth and plant biomass. The objective of this work was to discover a high-precision evaluation way of spraying cotton defoliating by comparing the multispectral remote sensing evaluation method with the traditional evaluation method. UAV multispectral images were collected in a cotton field of Yellow River Basin in China, and the images in five narrow bands included Red, Blue, Green, Near infrared (NIR) and Red Edge. The processing of multispectral images was performed in the Python environment, NDVI images and GNDVI images were created. Based on this, the NDVI data, GNDVI data and manual statistical data were compared. The results show that compared with traditional methods, the multispectral remote sensing evaluation method can be more accurate and effective in evaluating the spraying effect of cotton defoliant.
\end{abstract}

Keywords: multispectral remote sensing, defoliation, evaluation, precision agriculture UAV

DOI: $10.33440 /$ j.jpaa. 20210402.178

Citation: Zhang Y Z, Li W H, Ran W X, Gu J, Lan Y B. Comparative analysis of UAV RS and traditional cotton leaf falling evaluation methods. Int J Precis Agric Aviat, 2021; 4(2): 40-44.

\section{Introduction}

${ }^{1}$ Evaluating the spraying effect of defoliant is necessary condition to facilitate mechanized cotton harvesting. At present, manual calculation of cotton defoliation to evaluate the effect of spraying shedding is the main method in many studies. Zhu Jijie et al. studied the defoliant sensitivity of 12 different cotton varieties $^{[1]}$, while Gao Lili and Zou Xi et al. studied the application effect of different agents ${ }^{[2,3]}$. Ma Yan et al. sprayed defoliant in Xinjiang cotton fields with an unmanned aerial vehicle (UAV), and studied defoliant, wadding effect, and cotton quality ${ }^{[4]}$. Wang Yi et al. studied the effect of defoliation rate and yield of cotton after spraying defoliant ${ }^{[5]}$. The above experiments are to evaluate the effect of spraying and shedding by manually calculating the falling leaves of cotton ${ }^{[6]}$.

However, the above statistical techniques often cannot be effectively implemented for spatial data analysis because the techniques do not consider spatial connections or trends between data points (typically neighborhood points). For example, the traditional method to count the number of cotton leaves to evaluate

\section{Received data: 2021-09-12 Accepted data: 2021-10-20}

Biographies: Yuzi Zhang, Associate Professor of College of Agricultural Engineering and Food Science, Shandong University of Technology, P. R. China; research interests: agricultural automation and autonomy. Email: zhangyuzi@sdut.edu.cn; Wenhua Li, postgraduate, research interests: agricultural automation, Email: L17864306386@163.com; Weixu Ran, postgraduate, research interests: agricultural automation, Email: 625973407@ qq.com; Jian Gu, postgraduate, research interests: agricultural automation, Email: 625973407@qq.com.

* Corresponding author: Yubin Lan, Distinguished Professor, Director, research interests: precision agricultural aviation application,. Mailing Address: College of Electronic Engineering, South China Agricultural University. Email: ylan@scau.edu.cn. the efficiency of spraying cotton defoliating. Usually, five points method is popular in several experiments to save evaluation time. But however, the coverage of this method is narrow and the counting work is really complicated with not so reliable result. To overcome these shortages, a new evaluation method is proposed based on remote sensing to evaluate the result of spraying cotton defoliating ${ }^{[7,8]}$.

Recent years, remote sensing (RS) is becoming to be a crucial technology for the development of precision agriculture. Remote sensing technology has the potential to maximize the economic benefit and minimize environmental pollution through improved crop monitoring and management ${ }^{[9,10]}$. Airborne remote sensing offers flexibility for rapid image acquisition with high spatial resolution at different flight altitudes. Remote sensing technology, including hyperspectral, multispectral, thermal imaging and 3D LiDAR cloud point, provides some information such as Ratio Vegetation Index (RVI), Normalized Vegetation Index (NDVI), Difference Vegetation Index (DVI), Green Normalized Difference Vegetation Index (GNDVI) etc. to support the development of precision agriculture. Remote sensing data are widely used to classify crop types, and most crop type distribution maps is obtained by supervised classification methods $^{[11]}$. Recently, Unmanned Aerial Vehicle (UAV) Low Altitude Remote Sensing System has been applied widely, such as resources prospecting, disaster monitoring, because of its advantages in flexibility, economy, high efficiency, and real time monitoring $^{[12]}$.

From the aforementioned related work, it can be seen that: (1) The evaluation method of spraying and shedding effect is evaluated by manual calculation of cotton falling leaves. The statistical error is large, and the labor cost is high; (2) The analyzing methods: the traditional method of evaluating spraying by manually calculating the number of cotton falling leaves The method of 
defoliation effect will produce larger statistical errors and increase labor costs. Low-altitude remote sensing can accurately and timely estimate biophysical parameters, such as leaf area index (LAI), crop growth, and plant biomass. The objective and contribution of this work is to: collect multi-spectral images through drones, give different indicators such as NDVI and GNDVI, and analyze the defoliation effect of sprayed cotton. This method provides efficient and reliable analysis results for sprayed cotton defoliation ${ }^{[13]}$.

The rest of the paper is organized as follows. Section 2 describes the details of the proposed method to collect multispectral remote sensing data and generate the evaluation grid map. Section 3 describes the experiment results and data analysis. Section 4 gives the conclusion of this method ${ }^{[14]}$.

\section{Materials and methods}

\subsection{Instruments and equipment}

The cotton field is located at Wudi County, Binzhou City, in Shandong Province with planting mode "one film and three rows" in the saline and alkaline land. The equal row spacing of the cotton plants is around $76 \mathrm{~cm}$, while the average plant height is around $85 \mathrm{~cm}$. Weather condition were summarized as Table 1.

In this study, $360 \mathrm{~g} / \mathrm{L}$ thidiazuron $180 \mathrm{~g} / \mathrm{L}$ diuron suspension concentrate (Drop ultra ${ }^{\circledR}$ ) at 121.5 g a.i./ha (Hebei guoxinnonong Biotechnology Co., Ltd., Cangzhou, China) and 40\% ethephon at $480 \mathrm{~g}$ a.i./ha (September 22, 2018 was $600 \mathrm{~g}$ a.i./ha) (Jiangsu Anpon Electrochemical Co., Ltd., Huaian, China) were added into the tank. DJI T20 with six-rotor electric plant protection UAV with a maximum tank capacity of $20 \mathrm{~L}$ was used in the experiment ${ }^{[15]}$. This UAV supports autonomous obstacle avoidance and ground flight, with a working speed of $1-10 \mathrm{~m} / \mathrm{s}$, and an operating height of 1-30 m.

Table 1 The weather condition of test environment

\begin{tabular}{cccc}
\hline \multirow{2}{*}{ Item } & \multicolumn{3}{c}{ Data } \\
\cline { 2 - 4 } & 9.21 & 9.26 & 9.30 \\
\hline Temperature & $22^{\circ} \mathrm{C}-26^{\circ} \mathrm{C}$ & $20^{\circ} \mathrm{C}-25^{\circ} \mathrm{C}$ & $20^{\circ} \mathrm{C}-25^{\circ} \mathrm{C}$ \\
Humidity & $43.4 \%-54.5 \%$ & 47.4 to $62.5 \%$ & $38 \%-41 \%$ \\
Wind Speed & $0.40-1.15 \mathrm{~m} / \mathrm{s}$ & $0.31-1.23 \mathrm{~m} / \mathrm{s}$ & $0.8-1.3 \mathrm{~m} / \mathrm{s}$ \\
\hline
\end{tabular}

The DJI P4 Multispectral UAV was employed to take multispectral image of the cotton before and after spraying defoliating in the experiment. The UAV is flying with a certain trajectory as shown in Figure 1 and the multispectral camera is set to capture a picture every 3 minutes with $30 \mathrm{~m}$ flight. Available wavelengths $(\mathrm{nm})$ are Blue (450 nm center, $16 \mathrm{~nm}$ bandwidth), green (560 nm center, $16 \mathrm{~nm}$ bandwidth), red (650 nm center, $16 \mathrm{~nm}$ bandwidth), red edge (730 nm center, $16 \mathrm{~nm}$ bandwidth), near-IR (840 $\mathrm{nm}$ center, $26 \mathrm{~nm}$ bandwidth).

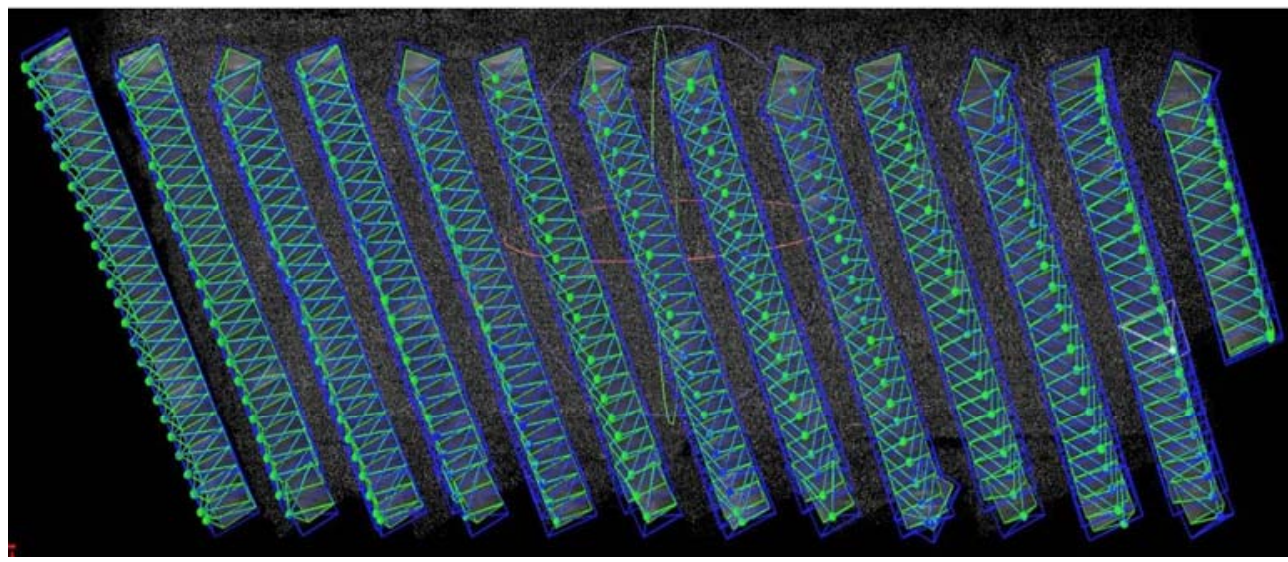

Figure 1 RS-UAV operation trajectory

2.2 Evaluation method of cotton defoliation rate based on UAV remote sensing image analysis

The images were collected in RGB and NIR bands through DJI P4 UAV at different stages before and after cotton spraying. These images were processed through different software. Python 3.7, software was used to process the UAV arthomosaic images of RGB and NIR. Firstly, a DJI P4 multispectral UAV was employed to capture the multispectral images of the operation area planted of cotton and hundreds of images were spliced to be a final image. Secondly, the normalized difference vegetation index (NDVI) and green light normalized difference vegetation index (GNDVI) of each period were analyzed by using python, and the cotton growth state map was constructed. Then, the predicted number of cotton defoliation in each period was retrieved, and combined with the quality of cotton defoliation, the evaluation method of cotton defoliation spraying accuracy based on multispectral remote sensing was constructed to estimate cotton defoliation.Finally, compared with the traditional method, the spraying effect was evaluated. The research scheme is shown in Figure 2. The detailed descriptions of this phase are illustrated as follows ${ }^{[16,17]}$.

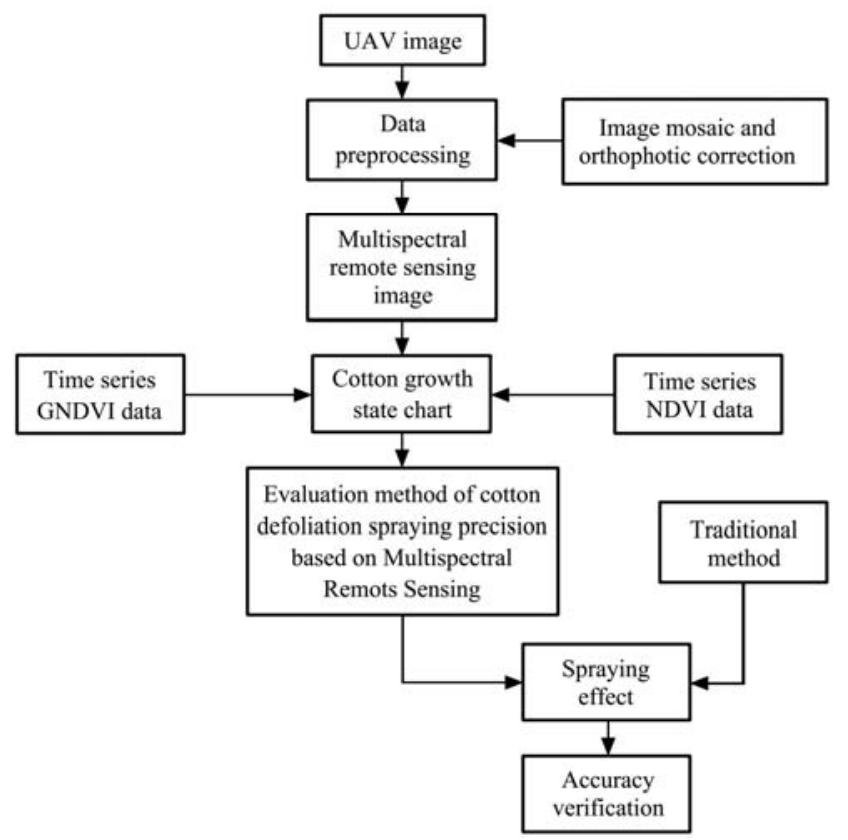

Figure 2 Technology roadmap 


\subsubsection{Evaluation Index}

The cotton defoliation rate was chosen as the field test targets to analyze the field efficacy of alkyl ethyl sulfonate dosage with different spray deposition characteristics. The number of leaves were investigated and recorded three times according to defoliant field efficacy test criteria (21st Sep2020, 26th Sep, 2020, 31st Sep 2020 and 7th Oct 2020). The defoliation rate was calculated using Equation (1):

$$
\text { Rate of defoliation (\%) }=\frac{N_{\mathrm{a}}-N_{\mathrm{b}}}{N_{\mathrm{a}}} \times 100 \%
$$

where, $N_{\mathrm{a}}$ is the number of leaves before treatment and $N_{\mathrm{b}}$ is the number of leaves after treatment.

According to the above formula, we employed more 5 students with 3 hours to count cotton leaves located at 5 different place. Each place, 5 different area was chosen. After that, all the data had to been keyed into the computer to plot the figures and tables. The results of artificial statistics of cotton defoliation are shown in Table 2.

Table 2 Number of leaves and defoliation rate corresponding to different days of each treatment

\begin{tabular}{ccccccc}
\hline & & \multicolumn{2}{c}{5 days after treatment } & & \multicolumn{2}{c}{10 days after treatment } \\
\cline { 3 - 4 } \cline { 6 - 7 } Treatment & $\begin{array}{c}\text { Number of } \\
\text { blades }\end{array}$ & $\begin{array}{c}\text { Number of } \\
\text { blades }\end{array}$ & $\begin{array}{c}\text { Deleafing } \\
\text { rate }\end{array}$ & & $\begin{array}{c}\text { Number of } \\
\text { blades }\end{array}$ & $\begin{array}{c}\text { Deleafing } \\
\text { rate }\end{array}$ \\
\hline 1 & 1180 & 889 & 24.70 & 562 & 52.40 \\
2 & 1196 & 879 & 26.50 & & 498 & 58.40 \\
3 & 1382 & 909 & 34.20 & & 424 & 69.30 \\
4 & 1339 & 969 & 28.40 & & 536 & 60.00 \\
5 & 1843 & 1297 & 29.60 & 673 & 63.50 \\
\hline
\end{tabular}

Table 3 shows the change rule of defoliation rate with time after spraying. It can be seen that the change trend of cotton defoliation rate with time was consistent in all treatments. The rate of defoliation increased rapidly within 5 days before defoliation and decreased after 5 days.

Considering that NDVI and GNDVI have the strongest ability to detect vegetation, these two vegetation indexes were used to calculate the vegetation coverage before and after cotton defoliation. The results are shown in Figure 3.

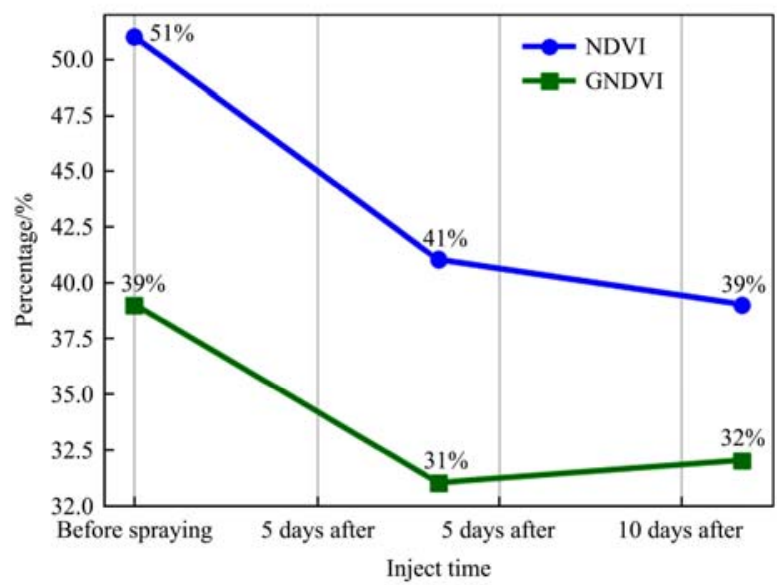

Figure 3 Changing Trend of NDVI and GNDVI

Figure 3 shows the change rule of defoliation rate with time after spraying. The rate of defoliation increased rapidly within 5 days before defoliation and decreased after 5 days. It can be seen from table 3 and Figure 3 that the results obtained by the two statistical methods are consistent, so the correlation between NDVI and GNDVI vegetation indexes and defoliation rate was the best. Therefore, NDVI and GNDVI were used as evaluation indexes to evaluate the effect of cotton defoliation.

Normalized Difference Vegetation Index (NDVI) is a popular index used to describe the crop grow conditions. The classical formula of NDVI is shown as follows,

$$
\mathrm{NDVI}=\frac{\mathrm{NIR}-\text { Red }}{\mathrm{NIR}+\text { Red }}
$$

where, NIR stands for near-infrared region 750-900 nm. The original NDVI of the cotton region in shown in Figure 4a and the grid map used to guide the spraying based on NDVI is shown in Figure 4b. Since the NDVI indicates the growth of cotton, it can be used to guide the spraying of defoliant. The higher value of NDVI, the more numbers of leaves of the cotton are growing well. In order to get good result of defoliant, the density of the spraying should be as high as possible.

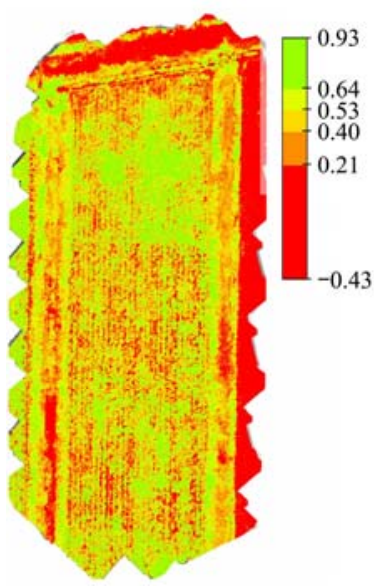

a.

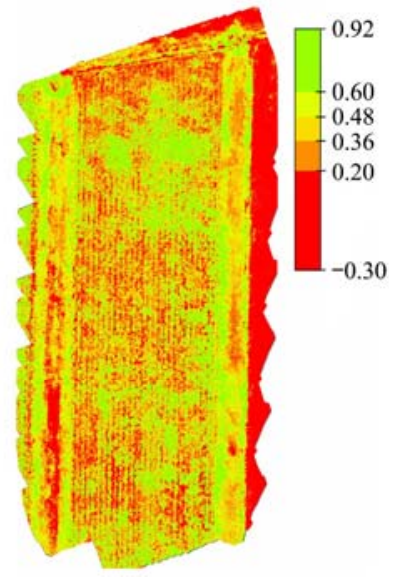

b.

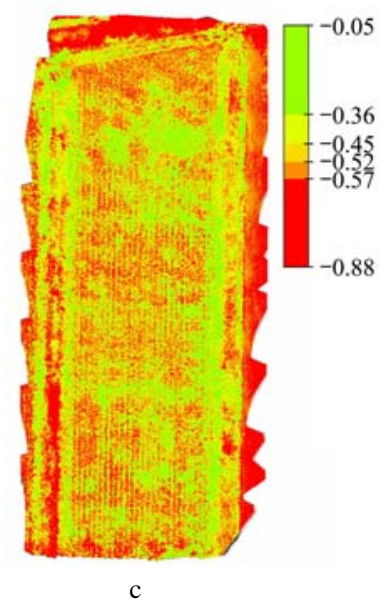

Figure 4 NDVI Image of different date after Spraying Cotton Defoliating deltofolin spraying

Except NDVI, Green NDVI (GNDVI) is also a classical index which is frequently used to describe the crop growth. The definition of GNDVI is defined as follows,

$$
\text { GNDVI }=\frac{\text { NIR }- \text { Green }}{\text { NIR }+ \text { Green }}
$$

The GNDVI was developed by ${ }^{[3]}$ using a green band in a study related to the remote sensing of global vegetation using EOSMODIS data. GNDVI is based on the idea that an index for chlorophyll estimation should be invariant with respect to pigments other than chlorophyll and should not be influenced by other factors. A curvilinear relationship between GNDVI and total chlorophyll content is reported in ${ }^{[4]}$.

2.1.2 Detection model of cotton defoliation rate

The different multispectral images collected on different date 
including 21st Sep2020, 26th Sep, 2020, 31st Sep 2020 and 7th Oct 2020 are shown in Figure 4a, b and c, respectively. NDVI and GNDVI were selected to establish the detection models of different multispectral vegetation index and cotton defoliation rate, and the different multispectral images collected on different dates were analyzed. Result and analysis are described in detail in Section 3.

\section{Results and analysis}

At present, there are two main ways of spraying cotton defoliant: average pesticide application and variable pesticide application. Average pesticide application refers to spray defoliant with same dose while the variable spraying application refers to spray defoliant with different dose. Here we use variable spraying to verify the grid map given by GNDVI or NDVI workable or not. In the experiment, the plant protection UAV of DJI T20 was used to carry out variable pesticide application by manually controlled in different working zone given by the grid map of GNDVI. Flying height of the UAV was $2 \mathrm{~m}$, the speed was $2 \mathrm{~m} / \mathrm{s}$, and the particle size of the fog droplet was 130 microns.

The number of leaves of the whole cotton plant that was numbered before application is investigated. The number of leaves remaining after application is reviewed at 5 days and 10 days, respectively. Results are summarized in Table 2.

From Table 3, it is easy to see that the original numbers of the whole cotton plant before spraying are proportional to the both the NDVI and GNDVI indexes. It means that the value of NDVI and GNDVI is bigger, the number of leaves is bigger.

Table 3 Results of the cotton defoliant experiments

\begin{tabular}{|c|c|c|c|c|c|c|c|c|c|c|c|c|}
\hline & \multicolumn{4}{|c|}{ Before spraying } & \multicolumn{4}{|c|}{5 days after } & \multicolumn{4}{|c|}{10 days after } \\
\hline & $\mathrm{LN}$ & $\mathrm{DR} / \%$ & NDVI & GNDVI & LN & $\mathrm{DR} / \%$ & NDVI & GNDVI & $\mathrm{LN}$ & $\mathrm{DR} / \%$ & NDVI & GNDVI \\
\hline 1 & 1180 & 21.2 & 0.48 & 0.51 & 889 & 24.7 & 0.41 & 0.59 & 562 & 52.4 & 0.45 & 0.62 \\
\hline 3 & 1382 & 31.2 & 0.49 & 0.63 & 909 & 34.2 & 0.37 & 0.56 & 424 & 69.3 & -0.55 & 0.54 \\
\hline 4 & 1339 & 24.1 & 0.60 & 0.69 & 969 & 28.4 & 0.45 & 0.62 & 536 & 60 & 0.42 & 0.62 \\
\hline 5 & 1843 & 26.2 & 0.59 & 0.67 & 1297 & 29.6 & 0.46 & 0.62 & 673 & 63.5 & 0.40 & 0.61 \\
\hline
\end{tabular}

\subsection{Efficiency comparison}

To further verify the effectiveness and advanced nature of cotton defoliation accuracy evaluation method based on multispectral remote sensing, the results of traditional method were used to verify the estimation results of this method.

Due to the limitation of the number of people and time, only five points are extracted from each region for manual statistics, so there was a large error. To solve this problem, the precision evaluation method of cotton defoliation spraying based on multispectral remote sensing and the " $\mathrm{X}$ " five point sampling method were used to evaluate the defoliation effect of cotton plants before and on the 5 and 10 days after application. As shown in Figure 5, the test field is divided into five processing areas, and then the data of each area is collected. The NDVI and GNDVI of each area are calculated by RS. The calculation results are shown in Table 4. Finally, the effect of defoliation was evaluated by NDVI and GNDVI.

Combined with Table 4, Figure 6 and Figure 7, it is easy to see that the original number of whole plant before spraying is directly proportional to NDVI index and GNDVI index. After spraying, NDVI and GNDVI values decreased with the change of spraying time, with the largest decline in the first 5 days and a smaller decline after 5 days, indicating that cotton defoliation rate increased rapidly in the first 5 days and decreased after 5 days.

Through the test results of traditional methods and multi spectral remote sensing based cotton defoliation spraying precision evaluation method, it can be seen that the cotton defoliation rate increased rapidly due to the high spraying concentration 5 days after spraying, and slowed down due to the low spraying concentration after 5 days spraying. Therefore, the analysis results of the two methods are consistent, which shows that the cotton defoliation spraying accuracy evaluation method based on multispectral remote sensing has high accuracy and credibility. In addition, as shown in Table 5, the proposed method of cotton defoliation spraying precision evaluation based on multispectral remote sensing can be completed by one person in 15 minutes compared with the traditional evaluation method. All data can be calculated by professional software, with high reliability.

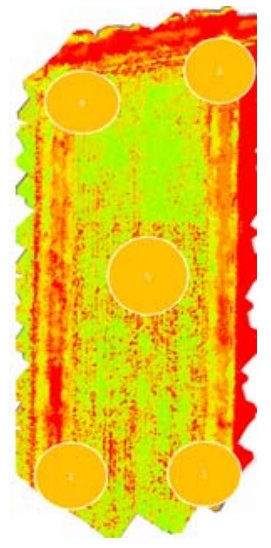

a.

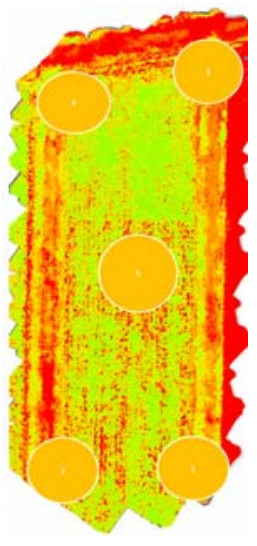

b.

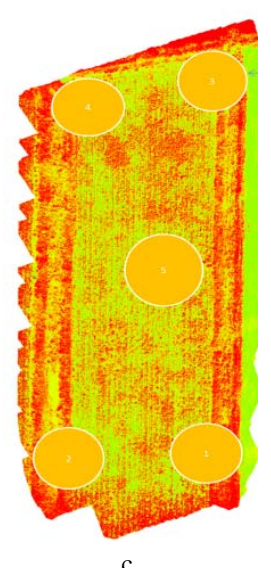

Figure 5 Location distribution of " $\mathrm{X}$ ” type five point sampling for cotton

Table 4 Results of the cotton defoliant experiments

\begin{tabular}{ccccccccc}
\hline & \multicolumn{2}{c}{ Before spraying } & & \multicolumn{2}{c}{5 days after } & & \multicolumn{2}{c}{10 days after } \\
\cline { 2 - 3 } \cline { 7 - 8 } \cline { 7 - 8 } & NDVI & GNDVI & & NDVI & GNDVI & & NDVI & GNDVI \\
\hline 1 & 0.59 & 0.67 & & 0.46 & 0.62 & & 0.45 & 0.62 \\
2 & 0.48 & 0.51 & & 0.41 & 0.47 & & 0.36 & 0.47 \\
3 & 0.33 & 0.53 & & 0.07 & 0.50 & & -0.05 & 0.49 \\
4 & 0.49 & 0.63 & & 0.37 & 0.56 & & 0.36 & 0.52 \\
5 & 0.60 & 0.69 & & 0.45 & 0.62 & 0.40 & 0.61 \\
\hline
\end{tabular}

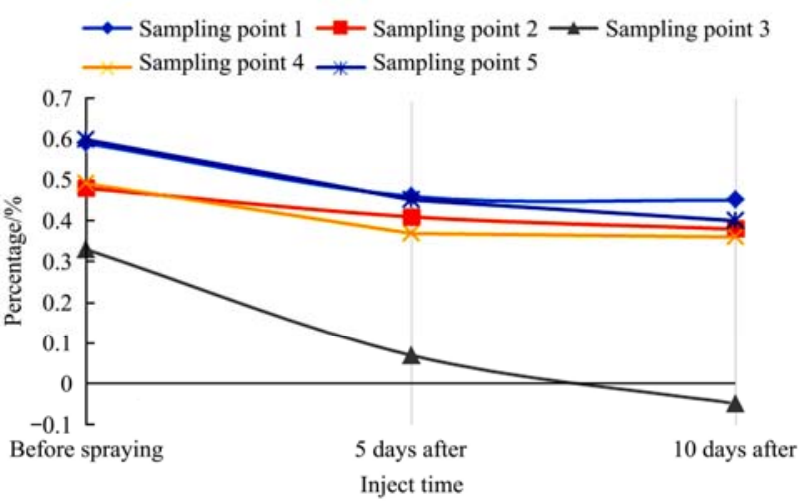

Figure 6 Changing Trend of NDVI 


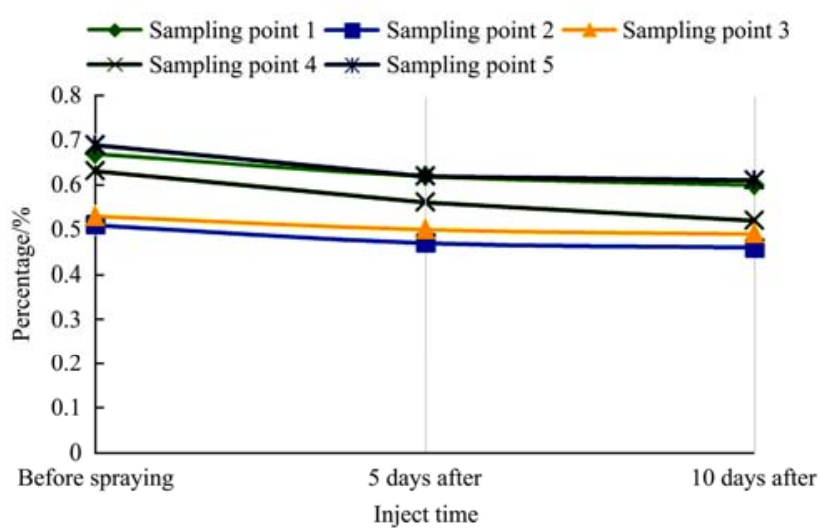

Figure 7 Changing Trend of GNDVI

Table 5 Comparison results of different methods

\begin{tabular}{lcc}
\hline \multirow{2}{*}{ Comparison parameters } & \multicolumn{2}{c}{ results of different methods } \\
\cline { 2 - 3 } & Traditional method & Proposed method \\
\hline Data processing time/h & 3 & 0.25 \\
Manpower required & 5 person & 1 person \\
Calculation error/\% & 5 & 0.8 \\
\hline
\end{tabular}

\section{Conclusions}

1) The multispectral based NDVI and GNDVI can be used to generate a grid map for guiding evaluating result of cotton defoliation since the relationship between the number of green leaves of cotton and the value of NDVI or GNDVI is simple proportional.

2) Compared with the traditional method, the proposed method can provide high efficiency and reliable result.

More UAV based remote sensing experiments on variable spraying, crop growth, monitoring of pests and diseases are planning to implement in the coming year.

\section{Acknowledgements}

We acknowledge that this work was financially supported by Top Talents Program for One Case One Discussion of Shandong Province, Program of Shandong Provincial Collaborative Innovation Center of Dry-farming Intelligent Agricultural Equipment, Academy of Ecological Unmanned Farm (2019ZBXC200) and China Agriculture Research System (CARS-15-22). We also thank the anonymous reviewers for their critical comments and suggestions to improve the manuscript.

\section{[References]}

[1] Zhu J J, Zhao H X, Wang S J, et al. Study on the sensitivity of different cotton cultivars to defoliant. China Cotton, 2018, 45(4): 15-18, 33. doi: 10.11963/1000-632X.20180416. (in Chinese)

[2] Zou X, Liu A Y, Li R L. Effects of different chemical ripening on disleaving and the yield and quality of cotton. Cotton Science, 2015, 37(4): 25-29. doi: 10.3969/j.issn.2095-3143.2015.04.006. (in Chinese)

[3] Ma Y, Ren X L, Meng Y H, et al. Review on result of spraying defoliant by unmanned aerial vehicles in cotton field of Xinjiang. China Cotton, 2016, 43(12): 16-20. doi: 10.11963/issn.1000-632X.201612004. (in Chinese)

[4] Gao L L, Li G, Xu X X, et al. Comparison of defolianting effects of the defoliants applied for 4 varieties of cotton. Journal of Xinjiang Agricultural University, 2016, 39(1): 35-39. (in Chinese)

[5] Wang Y, Yang L H, Shi L H. Effect of cotton defoliant on the defoliation rate and yield in Xinjiang. China Cotton, 2014, 41(4): 28-30. doi: 10.11963/issn.1000-632X.201512011. (in Chinese)

[6] E Raymond H, Caig D, Steven B M, W Dean H. Remote Sensing With Simulated Unmanned Aircraft Imagery for Precision Agriculture Applications. in IEEE Journal of Selected Topics in Applied Earth Observations and Remote Sensing, 2014, 7(11): 4566-4571. doi: 10.1109/JSTARS.2014.2317876.

[7] Huang Y, Lan Y F, Ge Y, et al. Spatial modeling and variability analysis for modeling and prediction of soil and crop canopy coverage using multispectral imagery from an airborne remote sensing system. Transactions of the ASABE, 2010, 53(4): 1321-1329. doi: 10.13031/ 2013.32582.

[8] C. Atzberger. Advances in Remote Sensing of Agriculture: Context Description, Existing Operational Monitoring Systems and Major Information Needs. Remote Sensing, 2013, 5(2): 949-981. doi: 10.3390/rs5020949.

[9] Li Y, Li Z, Tong L, Y, et al. A geometrical rectification algorithm of UAV remote sensing images based on flight attitude parameters. 2011 IEEE International Geoscience and Remote Sensing Symposium, 2011. doi: 10.1109/IGARSS.2011.6049171.

[10] Gitelson A A, Merzlyak M N, Lichtenthaler H K. Detection of red-edge position and chlorophyll content by reflectance measurements near $700 \mathrm{~nm}$. Journal of Plant Physiology, 1996, 148(3-4): 501-508. doi: 10.1016/ S0176-1617(96)80285-9.

[11] Shen X, Cao L, Liu K, She G H, Ruan H H. Aboveground biomass estimation in a subtropical forest using airborne hyperspectral data. 2016 4th International Workshop on Earth Observation and Remote Sensing Applications, 2016. doi: 10.1109/EORSA.2016.7552836.

[12] E Raymond H, Caig D, Steven B M, W Dean H. Remote sensing with unmanned aircraft systems for precision agriculture applications. 2013 Second International Conference on Agro-Geoinformatics, 2013. doi: 10.1109/Argo-Geoinformatics.2013.6621894.

[13] Bannari A, Karl S, Driss H, Shahid K K. Sensitivity Analysis of Chlorophyll Indices to Soil Optical Properties Using Ground-Reflectance Data. 2006 IEEE International Symposium on Geoscience and Remote Sensing Symposium, 2006. doi: 10.1109/IGARSS.2006.36.

[14] Andres V, Anatoly A G. Sensitivity to Foliar Anthocyanin Content of Vegetation Indices Using Green Reflectance. 2011 in IEEE Geoscience and Remote Sensing Letters, 2011. doi: 10.1109/LGRS.2010.2086430.

[15] Bannari A, Abdulaziz M, D. R. Peddle. Biophysiological spectral indices retrieval and statistical analysis for red palm weevil stressattack prediction using Worldview-3 data. 2016 IEEE International Geoscience and Remote Sensing Symposium, 2016. doi: 10.1109/IGARSS.2016.7729908

[16] Shen X, Cao L, Liu K, She G H, Ruan H H. Aboveground biomass estimation in a subtropical forest using airborne hyperspectral data. 2016 4th International Workshop on Earth Observation and Remote Sensing Applications, 2016. doi: 10.1109/EORSA.2016.7552836

[17] Wen S, Han J, Lan Y B, et al. Influence of Wing Tip Vortex on Drift of Single Rotor plant protection unmanned aerial vehicle. Transactions of the Chinese Society for Agricultural Machinery, 2018, 49(8): 127-137. doi: 10.6041/j.issn.1000-1298.2018.08.015.

[18] Xu B, Chen L P, Xu M, et al. Path planning algorithm for plant protection UAVs in multiple operation areas. Transactions of the Chinese Society for Agricultural Machinery, 2017, 48(2): 75-80. dio: 10.6041/ j.issn.1000-1298.2017.02.010.

[19] Qin W C, Xue X Y, Cui L F, et al. Optimization and test for spraying parameters of cotton defoliant sprayer. Journal of Chinese Agricultural Mechanochemistry, 2017, 38(4): 25-32. dio: 10.3965/j.ijabe.20160904. 2125.

[20] Peter W P, Gerald M. L. Effects of diuron and other aerially applied cotton herbicides and defoliants on the plankton communities of aquaculture ponds, 2004, 233: 197-203. dio: 10.1016/j.aquaculture.2003. 09.029. 